\title{
Resolving Nanoscale Heterogeneity in Battery Interphases with Cryo-EM
}

William Huang ${ }^{1}$, Hansen Wang ${ }^{2}$, David Boyle ${ }^{2}$, Yuzhang $\mathrm{Li}^{2}$ and Yi Cui ${ }^{2}$

${ }^{1}$ Stanford University, Palo Alto, California, United States, ${ }^{2}$ Stanford University, Stanford, California, United States

Lithium (Li)-based batteries are critical to the decarbonization of the transportation sector owing to their high energy densities [1]. However, batteries are notoriously complex, and a nanoscopic understanding of the structure and function of the various interfaces and interphases within the battery remains elusive. Most prominent among these battery structures is the solid-electrolyte interphase (SEI), a passivation layer that exists on the negative electrode (anode) of the battery [2]. This SEI has been referred to as the "most important and the least understood" aspect of the battery [3], as it dictates the stability and cyclability of the negative electrode, while remaining resistant to nanoscale interrogation by electron-optical methods such as transmission electron microscopy (TEM) as a result of their sensitivity to air, moisture, and electron irradiation. Surface-analytical techniques with poor in-plane resolution such as x-ray photoelectron spectroscopy (XPS) have thus become standard analytical techniques for the study of SEI, and anode stability is often attributed to the presence of SEI components rather than their distribution.

Owing to recent developments in cryogenic TEM for materials science, it becomes possible to image Libased battery materials in the TEM without side reactions and beam damage, and the spatial distribution of SEI components across the anode becomes resolvable [4,5,6]. Using cryogenic scanning TEM (cryoSTEM) coupled with electron energy loss spectroscopy (EELS), we map the distribution of SEI species across the metallic $\mathrm{Li}$ anode, covering length scales from the nanoscale to mesoscale. We find that $\mathrm{LiF}$, an SEI component believed to be indispensable to the anode performance [7], lies outside of the "compact" SEI covering the Li metal surface, which is comprised primarily of carbonates and oxides (Fig. 1). Instead, LiF precipitates across the electrode as discrete nanoparticles (Fig. 2). LiF, thought to be a critical component within the compact SEI owing to its passivating nature, likely plays a more nuanced role in the SEI. The use of high spatial resolution techniques such as Cryo-(S)TEM reveals the heterogeneous nature of SEI between the atomic and electrode length scales, which is not observable through surface analytical methods. This work bridges the gap between microscale characterizations of battery electrodes and atomic scale characterizations, revealing the nanoscale and mesoscale heterogeneity of battery interphases. 
a
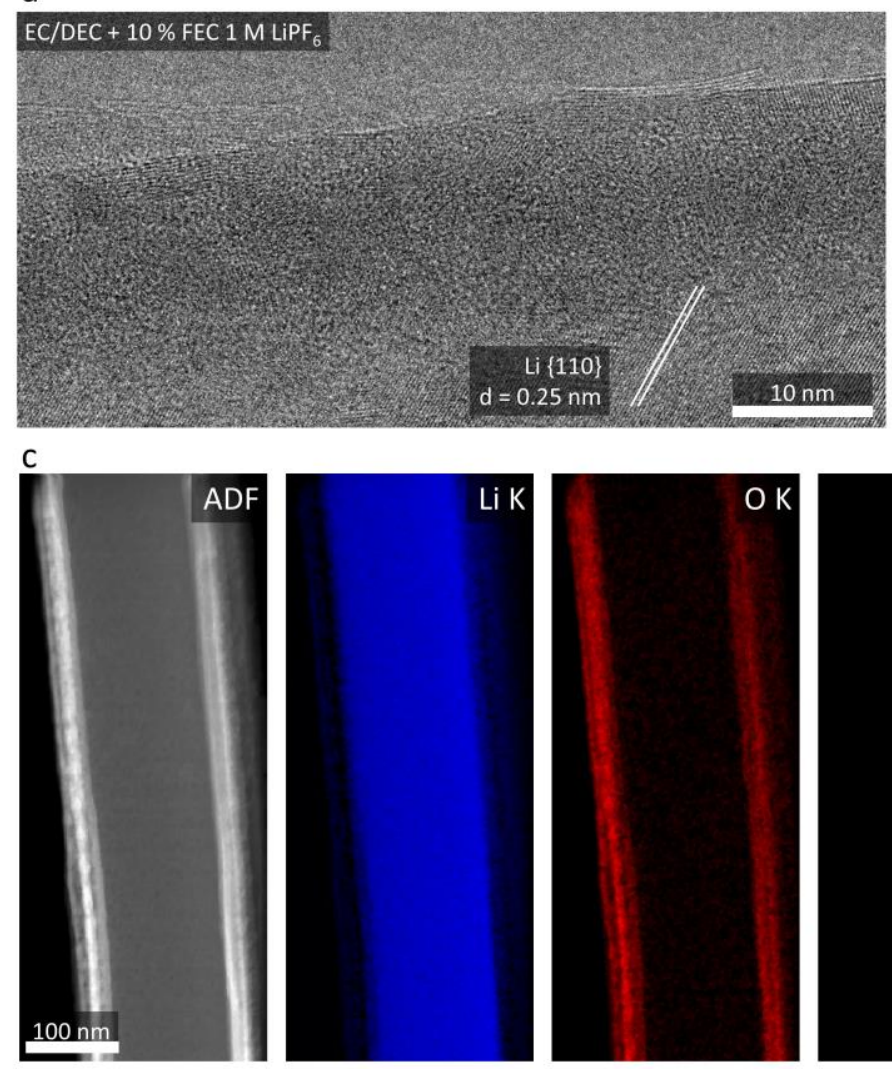

Figure 1. Cryo-(S)TEM characterizatio of Li metal and its compact SEI in a fluorinated electrolyte. (a) Cryo-HRTEM of the compact SEI directly interfaced with Li metal in EC/DEC 10\% FEC 1.0 M LiPF6 electrolyte. The image is $60 \mathrm{~nm}$ wide. (b) Fast Fourier transform (FFT) of 1d. Reflections are matched to $\mathrm{Li} 2 \mathrm{O}$ and $\mathrm{Li}$, without any $\mathrm{LiF}$ reflections present. (c) Cryo-STEM EELS mapping of $\mathrm{Li}, \mathrm{O}, \mathrm{F}$ of a representative Li metal filament region without any F signal. Window is $270 \mathrm{~nm}$ x $640 \mathrm{~nm}$.
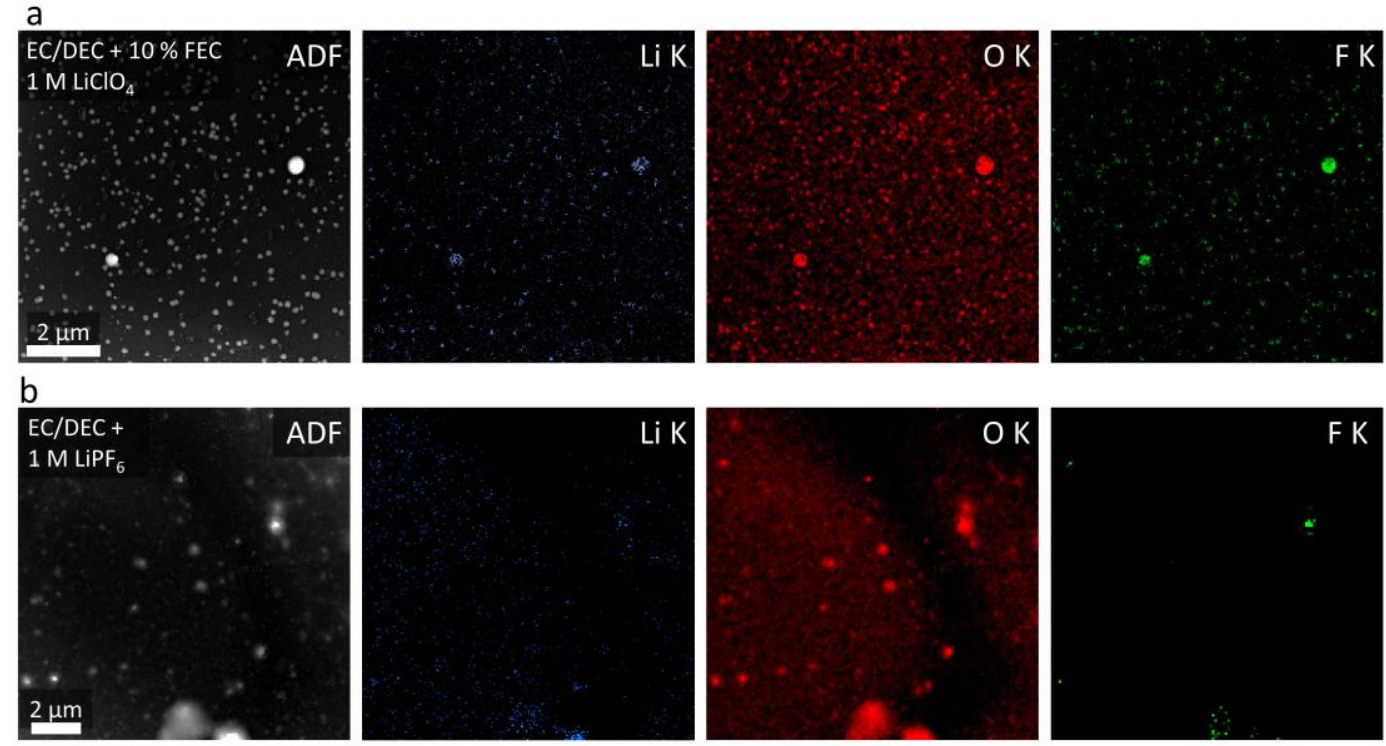

Figure 2. Cryo-STEM EELS mapping of the SEI formed on an inert, flat substrate. (a) Li, O, F mapping in a highly fluorinated electrolyte. (b) Li, O, F mapping in a standard electrolyte. 


\section{References}

(1) Chu, S.; Cui, Y.; Liu, N. The Path towards Sustainable Energy. Nat. Mater. 2016, 16 (1), 16-22.

(2) Peled, E. The Electrochemical Behavior of Alkali and Alkaline Earth Metals in Nonaqueous Battery Systems-The Solid Electrolyte Interphase Model. J. Electrochem. Soc. 1979, 126 (12), 2047.

(3) Winter, M. The Solid Electrolyte Interphase - The Most Important and the Least Understood Solid Electrolyte in Rechargeable Li Batteries. Zeitschrift fur Phys. Chemie 2009, 223 (10-11), 1395-1406.

(4) Zachman, M. J.; Tu, Z.; Choudhury, S.; Archer, L. A.; Kourkoutis, L. F. Cryo-STEM Mapping of Solid-Liquid Interfaces and Dendrites in Lithium-Metal Batteries. Nature 2018, 560 (7718), 345-349.

(5) Li, Y.; Li, Y.; Pei, A.; Yan, K.; Sun, Y.; Wu, C. L.; Joubert, L. M.; Chin, R.; Koh, A. L.; Yu, Y.; et al. Atomic Structure of Sensitive Battery Materials and Interfaces Revealed by Cryo-Electron Microscopy. Science. 2017, 358 (6362), 506-510.

(6) Wang, X.; Zhang, M.; Alvarado, J.; Wang, S.; Sina, M.; Lu, B.; Bouwer, J.; Xu, W.; Xiao, J.; Zhang, J. G.; et al. New Insights on the Structure of Electrochemically Deposited Lithium Metal and Its Solid Electrolyte Interphases via Cryogenic TEM. Nano Lett. 2017, 17 (12), 7606-7612.

(7) Fan, X.; Ji, X.; Han, F.; Yue, J.; Chen, J.; Chen, L.; Deng, T.; Jiang, J.; Wang, C. Fluorinated Solid Electrolyte Interphase Enables Highly Reversible Solid-State Li Metal Battery. Sci. Adv. 2018, 4 (12). 